\title{
LA RELECTURA DE LA HISTORIA, UN ARMA DE LUCHA DE LAS MUJERES INDÍGENAS
}

\section{THE REREADING OF HISTORY, A WEAPON OF INDIGENOUS WOMEN}

\author{
Erika Paola Serrano Rojas ${ }^{(1)}$ \\ Universidad Autónoma de Madrid, España \\ Universidad Nacional San Antonio Abad del Cusco, Perú
}

\begin{abstract}
Resumen: En las últimas décadas el feminismo en América Latina ha tomado un nuevo curso. Gracias a los aportes de trabajos críticos y de reflexión de numerosas autoras se visualizan nuevos retos que trascienden el carácter limitado y excluyente de los conceptos establecidos por un feminismo hegemónico. La lucha por la reivindicación de las mujeres y las mujeres indígenas en América Latina no puede ser entendida y llevada según los lineamientos establecidos por occidente. El presente artículo pretende resaltar el entendimiento del feminismo surgido en América Latina y el feminismo comunitario a partir del libro "Hilando Fino desde el feminismo comunitario" de Julieta Paredes, del cual extraigo una propuesta concreta la relectura de la historia como un arma de lucha para mujeres de comunidades indígenas, que tiene el reto de replantear la exigencia de los derechos de las mujeres indígenas a partir de su cultura.
\end{abstract}

Palabras clave: feminismo hegemónico, reivindicación, coyunturas, comunidades indígenas, feminismo comunitario, relectura de la historia, cultura.

Summary: In recent decades feminism in Latin America has taken a new course. Thanks to the contributions of critical works and reflection of numerous authors, new challenges are visualized that transcend the limited and exclusive nature of the concepts established by a hegemonic feminism. The struggle for the vindication of indigenous women in Latin America cannot be understood and carried out according to the guidelines established by Occident. This article intends to highlight

(1) Magister en Antropología de Orientación Pública por la Universidad Autónoma de Madrid, España. Licenciada en Antropología en la Universidad Nacional San Antonio Abad del Cusco, Perú. Especialista en proyectos sociales y gestión cultural. email: erika.serrano@unsaac.edu.pe 
important issues that will bring us closer to the understanding of feminism that has emerged in Latin America and community feminism from the book "Hilando Fino from feminist feminism" by Julieta Paredes, from which I extract a specific proposal rereading of history as a weapon of struggle for women from indigenous communities, for rethinking the demand for the rights of indigenous women based on their culture. 


\section{Introducción}

El feminismo ha tenido una gran aportación para visibilizar los derechos subordinados de las mujeres. En sus inicios, este feminismo se ha desarrollado y ha respondido a las necesidades y contexto de un grupo de mujeres pertenecientes al mundo europeo - occidental. Sin embargo, gracias a un análisis crítico por parte de numerosas intelectuales y activistas, se ha desarrollado nuevos debates en busca de la igualdad de las mujeres y sus derechos en todos los ámbitos. Es decir, este movimiento universal surgido de la ilustración en Europa fue progresivo, y de la mano con la modernidad resurge en Estados Unidos para cuestionarse la discriminación del propio feminismo hacia las mujeres negras, y luego expandir esta cuestión critica al resto del mundo no occidental, llegando a América Latina como una revolución cultural. Un proceso que en la actualidad se enmarca en la reinterpretación de aquel discurso hegemónico surgido en Europa, con el fin de incluir la voz de las mujeres indígenas. La atención del presente artículo se centra en realizar una breve aproximación al desarrollo del feminismo comunitario, a partir del Libro "Hilando fino desde el feminismo comunitario" propuesto por Julieta Paredes y entender que esta iniciativa latinoamericana necesita armas de lucha en sus propios contextos.

\section{El feminismo y el feminismo comunitario}

La historia nos relata que el "feminismo es hijo no querido de la ilustración" (Valcárcel, 2008), surgioa en un contexto europeo que responde a las necesidades propias de una determinada realidad y época. Un proceso que se ha ido afirmando socio políticamente en la búsqueda de la reivindicación del papel de la mujer en la sociedad y la vida. Sin embargo, en un mundo de diversas realidades, culturas y contextos, esta búsqueda de la reivindicación de los derechos de la mujer supone diferentes perspectivas y líneas de intervención, que posteriormente y de manera paulatina han sido abordados.

El feminismo surgido en América Latina estuvo marcado por diferentes dinámicas sociales, políticas y económicas (Schild, 2016), correspondientes a países con dictaduras y conflictos armados internos como es el caso de Guatemala, Nicaragua, Perú y Colombia, donde el papel de la mujer en la coyuntura política y social ha sido importante (Rivera, 2012). Así mismo, en un contexto capitalista y moderno, la emancipación de la mujer en América Latina estuvo considerada en función a su participación en el mercado, surgiendo así un movimiento feminista que se desarrolló en un ámbito urbano dirigido y pensado por mujeres de clase media urbana con acceso a la educación (Suárez, 2012). Una situación que encubría las relaciones jerárquicas internas de clase, raza y cultura de las demás mujeres que vivían en el país, suponiendo de esta manera la ausencia de las mujeres indígenas en estos espacios. 
Evidentemente, el feminismo hegemónico definió los lineamientos de lucha de las mujeres en América Latina mediante enfoques que no solo fueron liberales sino también coloniales, subsumiendo en un solo discurso las mismas reivindicaciones para todas las mujeres. Sin embargo, es en la década de los 90 cuando las voces indígenas comienzan a visibilizar la ideología limitada y excluyente desarrollada por el feminismo hegemónico (Galván, 1995). De esta manera, se genera una preocupación que hace repensar el feminismo en el contexto de la auto representación indígena, mostrando no solo un sistema patriarcal de opresión sino también una suerte de colonización y dominación cultural; donde de la mujer indígena se ubicaba en el último escaño de las clases sociales (Gargallo, 2014).

Para Julieta Paredes la construcción y comprensión del feminismo comunitario implica partir en primera instancia de la idea de que "los feminismos comparten el mismo origen, convicciones y principios fundamentales" y que el feminismo "hegemónico" u occidental ha aportado categorías indispensables en el entendimiento de la realidad de la mujer. En otros términos Espinosa amplia esta visión en la misma tendencia y expone el planteamiento de dos ejes fundamentales sobre el que se levanta cualquier feminismo: el primero, explica aquella idea compartida sobre la situación de subordinación y dominación de las mujeres dentro de un sistema estructural que pone al hombre al frente del control de las instituciones, la construcción del saber y la capacidad de reproducción de la mujer, y el segundo trata sobre la necesidad histórica de la aparición del feminismo como movimiento social que aglutina a las mujeres a rebelarse contra el sistema (Espinoza. 2008).

En esta línea, otro aporte singular está referido a la categoría de género y al entendimiento de la opresión y el sistema patriarcal (Paredes, 2010). El primero expresa un concepto cuyo proceso histórico ha logrado visibilizar las desigualdades sociales de la mujer, contribuyendo al análisis crítico de las relaciones sociales que se establecen en una realidad de dominación patriarcal; lo que a su vez hace repensar la situación de la mujer indígena. Un aporte que generaría a su vez categorías autónomas identificadas a partir de la realidad cultural de las comunidades indígenas. En síntesis, como ya se dijo, la propuesta del feminismo comunitario evoca la construcción de líneas de análisis para la opresión histórica cultural que han sufrido las mujeres indígenas partiendo y reconociendo la importancia de los aportes occidentales, en la búsqueda de delimitar su acción frente a un sistema de dominación patriarcal.

De esta manera, según Paredes es indispensable el reconocimiento y crítica a las categorías modernas occidentales con la finalidad de plantear un desenganche epistemológico y al mismo tiempo proponer otras categorías analíticas que respondan a contextos indígenas. Esto permitiría fortalecer las prácticas políticas de la trasformación social para las comunidades indígenas en 
América Latina. Finalmente, de lo señalado, no se trata de inferir una disputa o enfrentamiento entre el feminismo hegemónico y el feminismo comunitario. Y mucho menos tratar de sobreponer una corriente sobre otra, sino más bien se trata de entender y trascender la idea de un feminismo único de reivindicación e igualdad, puesto que la lucha que incluye a todos los sectores e implica a su vez la cuestión de la mujer indígena.

\section{El feminismo se asume dentro de una cultura que tiene que buscar sus propias armas de lucha contra el sistema patriarcal}

Difícilmente podríamos imaginar un feminismo comunitario sin los aportes sustanciales que brindó el movimiento zapatista en México. Este movimiento jugó un papel importante en la creación de espacios de reflexión y organización para las mujeres indígenas, visibilizando sus demandas. Sin embargo, no es posible entender la fuerza de los movimientos de mujeres indígenas, sin considerar sus experiencias y protagonismo a lo largo de la historia de las naciones y pueblos. (Hernàndez, 2012 ).

La lucha de las mujeres se produce y no se limita al ámbito de los grandes acontecimientos políticos o de las grandes decisiones económicas e ideológicas (Figueroa, 2013)."El feminismo es la lucha y la propuesta política de vida de cualquier mujer en cualquier lugar del mundo y en cualquier etapa de la historia que se haya rebelado ante el patriarcado que las oprime" (Paredes, 2010). Cada sociedad a su medida ha buscado las armas para defenderse o tal vez sobrellevar las diferencias. América latina, con sus diferentes culturas y contextos cuenta con una particularidad histórica propia, pues presenta una lucha constante por la reivindicación de los pueblos originarios y dentro de ésta, la búsqueda de la reivindicación de la mujer indígena. Un proceso invisibilizado poco estudiado y conocido dentro del cual las mujeres indígenas se han enfrentado no solamente al racismo, la exclusión social de un sistema doblemente marcado por el patriarcado, sino también los estereotipos sociales, económicos y culturales que las mujeres blancas han manejado en dicho sistema.

Frente a este contexto la respuesta de las mujeres indígenas se ha producido combinando luchas contra varias formas de desigualdad y opresión. Una lucha desarrollada en diversos momentos que ha tenido dificultades para tener consciencia y lograr una dirección autónoma sin influencias externas, o discursos esencialistas. Por lo tanto retomando lo mencionado anteriormente no solo se trata de encontrar categorías autónomas a nuestras realidades sino también es necesario conocer los mecanismos políticos, económicos e ideológicos que han experimentado las mujeres indígenas y sus respectivas respuestas, reconociendo el aporte y el papel que han jugado en sus propias culturas. 


\section{Una relectura de la historia mujeres con derecho a ser las protagonistas}

Una revisión histórica o mejor dicho una relectura de la historia en términos de Ochy Curiel es clave para repensar la dirección del feminismo aplicado a Latinoamérica (Curiel, 2017). Muchísimos son los ejemplos de personajes célebres que aportaron en la construcción de una sociedad más justa para la mujer, por lo que es trascendental "comprender su lugar en la sociedad, su condición, sus papeles, su poder, su silencio y su palabra" (Duby y Perrot,1993)

En esta parte del artículo mostraré ejemplos interesantes que muestran las distintas armas de mujeres indígenas peruanas que se desarrollaron dentro de una historia marcada por el colonialismo. Un sistema en el que, según Tiitinen, se entrecruzan diferentes dimensiones de la identidad en la mujer indígena, categorizando tres espacios de marginación: primero por ser mujer, el segundo por ser indígena y por el último la clase a la que pertenecen "pobres" (Tiitinen, 2011).

Margarita de Ayala, la india Pretona García, Leboria Barba y sus hijas, Manuela Rodríguez entre otras son referencia de mujeres indígenas en el departamento de Lambayeque, Perú (Figueroa,2013), quienes entre los años 1780-1850, de acuerdo a su contexto y situación, establecieron mecanismos de defensa, resistencia y cambio frente a un sistema de opresión. En este sentido, con el fin de rastrear la historia escondida y silenciada, Walker y Stern categorizaron las resistencias de opresión en 3 tipos de respuestas (Walker y Stern,1990)

- La colaboración o cooptación, cuando las víctimas apoyan a los oprimidos con el interés de lograr una ventaja.

- La resistencia, cuando se busca eliminar la situación de opresión

- La adaptación, en sus dos subtipos: adaptación pasiva, que es la resignación y adaptación en resistencia que buscaría el menor grado de opresión

Estos ejemplos muestran según Figueroa una lucha desarrollada en diversos momentos y condiciones, en los cuales las mujeres indígenas en ocasiones tuvieron que aceptar una situación de extrema desigualdad y opresión de género pasando a la resistencia en forma de autodefensa como la fuga o procedimientos legales. Una forma destacada de adaptación en resistencia fue la solidaridad con los presos, perseguidos y víctimas de abusos. Por otro lado, cuando la resistencia contra la opresión social y colonial se agudizó, las mujeres indígenas no solo participaron junto con los varones en la lucha por la libertad e igualdad, sino que compartieron con ellos el liderazgo, como viene a ser el caso de Micaela Bastidas. 
Una relectura de la historia significa cambiar todo un andamiaje de ideas y creencias para transformar las actividades femeninas en experiencias definidas y trascendentes que sean valoradas en el curso del desarrollo de la humanidad, la cultura y la civilización. Situar la lucha desde los espacios comunitarios implica una lógica de investigación diferente a la aplicada en la historiografía tradicional es decir reescribir la historia desde una alternativa contestataria con nuevos modelos interpretativos para afirmar los procesos de búsqueda de la reivindicación de la mujer indígena a fin de fortalecer desde las bases una propia lógica de lucha. (Joan Scott, 1993).

\section{Conclusiones}

El análisis crítico del origen del feminismo, sus principios comunes y aportes de categorías que visibilizaron la desigualdad de género, hacen posible la reconceptualización de nuevas propuestas. En esta nueva percepción de lo que implica el feminismo comunitario, los términos y la trascendencia de categorías y conceptos se convierten en una herramienta eficaz para la investigación de los procesos socio-históricos en América Latina. Realizar una reconstrucción de la historia de la mujer y mujeres indígenas, con sus aportaciones en la autonomía feminista, es un arma de empoderamiento y lucha para el ejercicio pleno de sus derechos.

En la actualidad, el feminismo comunitario es una iniciativa que se encuentra en construcción desde la visión de mujeres de diversos contextos y culturas quienes encuentran sus propias armas de lucha. Por décadas, en las comunidades ha existido una suerte de encubrimiento, parte de la ideología feminista se volvió "ongeinista" (de ONG) o esencialista, por lo que a partir de un análisis crítico de categorías y una relectura de la historia se enmarca en una propuesta autónoma, que implica otras formas de conocimientos y producir pensamientos con el fin de construir la tan anhelada igualdad de oportunidades y derechos de las mujeres indígenas.

\section{Bibliografia}

CURIEL, O. (2017) Las Claves de Ochy Curiel. Feminismo Decolonial. Agencia Andaluza de Cooperación Internacional para el Desarrollo (AACID). https://www.youtube.com/watch?v=7ZSHqvKLANQ\&t=538s

DUBY Y PERROT M. en Garcia J., (1993) Historia de las mujeres en América latina enfoques renovados y urgentes necesidades. Historia de las mujeres en América Latina. Centro de Estudios la mujer en la historia de América Latina, CEMHAL. pág. 14 
ESPINOSA, Y. (2008), Critica epistémica y feminismo decolonial. Conferencia en Universidad Autónoma de recuperado 01 de octubre del 2013. México.

https://www.youtube.com/watch?v=HnjXiWIFSX0\&t=96s

FIGUEROA, G. (2013). La mujer lambayecana en la lucha social y anticolonial 17801850 en Andreo, J. y Guardia, S. Historia de las mujeres en América Latina. Centro de Estudios la mujer en la historia de América Latina, CEMHAL. pág. 150.

GALVÁN, S. (1995). El mundo étnico racial dentro del feminismo latinoamericano, Cuadernos África América Latina, http://publicaciones.sodepaz.org/images/uploads/documents/revista019/04_mundoetnico.pdf

GARGALLO. F. (2013). Feminismos desde Abya Yala. Ideas y Proposiciones de las mujeres de 607 Pueblos en nuestra América. Cuidad de México. Editorial Corte y Confección,.pag. 26

HERNANDEZ. A. (2012). Entre el Etnocentrismo feminista y el esencialismo étnico de las mujeres indígenas y sus demandas de género. Entre el etnocentrismo feminista y el esencialismo étnico, Debate Feminista Vol. 24 Octubre

JOAN SCCOT, (1993) Historia de las mujeres en Burke, P. Formas de hacer historia, en Andreo J. Historia de las mujeres en América latina enfoques renovados y urgentes necesidades. Pág. 59- 88

PAREDES, J. (2010). Hilando Fino. Desde el Feminismo Comunitario. la Paz: Coopertiva El Rebozo

RIVERA, T. Mujeres indígenas Americanas luchando por sus derechos en Suarez, L. y Hernández, R. Descolonizando el feminismo teorías y prácticas desde los márgenes. pag. 329

SCHILD, V. (2016). Feminismo y neoliberalismo. Nueva Sociedad Democracia y Política en América Latina. Recuperado 20 de diciembre 2017 http://nuso.org/articulo/feminismo-y-neoliberalismo-en-america-latina/

STERN, S. (1990). Resistencia, Rebelión y Conciencia Campesina en los Andes. en Figueroa G. (2013). La mujer lambayecana en la lucha social y anticolonial 17801850 en Andreo, J. y Guardia, S. Historia de las mujeres en América Latina. Centro de Estudios la mujer en la historia de América Latina, CEMHAL. pág 152:

TIITINEN V. (2011). Feminismo indígena de un análisis Crítico del discurso sobre los textos de la mujer en el movimiento zapatista 1994- 2009, helsinki. pag 254

VALCÁRCEL, A. (2008); ¿Qué es el Feminismo? La metáfora de las gafas violetas, en N. Valera; Feminismo para principiantes. Pág.10. 